\title{
Papers
}

\section{The Lambeth Early Onset (LEO) Team: randomised controlled trial of the effectiveness of specialised care for early psychosis}

Tom K J Craig, Philippa Garety, Paddy Power, Nikola Rahaman, Susannah Colbert, Miriam Fornells-Ambrojo, Graham Dunn

\begin{abstract}
Objective To evaluate the effectiveness of a service for early psychosis.

Design Randomised controlled clinical trial.

Setting Community mental health teams in one London borough.

Participants 144 people aged 16-40 years presenting to mental health services for the first or second time with non-organic, non-affective psychosis.

Interventions Assertive outreach with evidence based

biopsychosocial interventions (specialised care group) and standard care (control group) delivered by community mental health teams.

Primary outcome measures Rates of relapse and readmission to hospital.

Results Compared with patients in the standard care group, those in the specialised care group were less likely to relapse (odds ratio $0.46,95 \%$ confidence interval 0.22 to 0.97 ), were readmitted fewer times ( $\beta 0.39,0.10$ to 0.68$)$, and were less likely to drop out of the study (odds ratio $0.35,0.15$ to 0.81 ). When rates were adjusted for sex, previous psychotic episode, and ethnicity, the difference in relapse was no longer significant (odds ratio $0.55,0.24$ to 1.26 ); only total number of readmissions ( $\beta 0.36,0.04$ to 0.66$)$ and dropout rates $(\beta 0.28$, 0.12 to 0.73 ) remained significant.

Conclusions Limited evidence shows that a team delivering specialised care for patients with early psychosis is superior to standard care for maintaining contact with professionals and for reducing readmissions to hospital. No firm conclusions can, however, be drawn owing to the modest sample size.
\end{abstract}

\section{Introduction}

Worldwide there is interest in developing early intervention services for people with a first psychotic disorder. Fifty such services are to be established in England. ${ }^{12}$ The rationale behind these services is that a long duration of untreated psychosis may have a detrimental effect on outcome and that sustained treatment in the two or three years after an acute episode should maintain recovery and rebuild careers, relationships, and leisure pursuits. ${ }^{3-10}$ Although the rationale may be compelling, only one randomised controlled trial has studied early intervention in patients with a first episode of psychosis. ${ }^{11}$ Other evidence comes from studies using historical controls or specific interventions such as atypical antipsychotics, cognitive behavioural therapy, and family therapy. ${ }^{4}{ }^{12-21}$
We investigated whether a specialist team could achieve better outcomes for people with early non-affective psychotic disorders than existing services. We hypothesised that, over an 18 month period, people receiving specialised care would have more frequent contact with mental health services, fewer relapses, and fewer readmissions to hospital than patients receiving standard care.

\section{Methods}

We considered all people aged 16-40 years living in the London borough of Lambeth and presenting to mental health services for the first time with non-affective psychosis (schizophrenia, schizotypal, and delusional disorders, F20-29; international classification of diseases, 10th revision). We also considered people who had presented once but had subsequently disengaged without treatment from routine community services. We excluded those with organic psychosis or a primary alcohol or drug addiction. Non-English speakers were not excluded, but we did exclude asylum seekers who were liable to enforced dispersal.

Lambeth is the seventh most deprived of the 376 local authority boroughs in England and Wales. ${ }^{22}$ It has a sizeable population from ethnic minority groups and unemployment is around twice the national average (2001 census). Community mental health services are provided through five multiprofessional teams.

\section{Interventions}

Assertive outreach for early psychosis

The Lambeth Early Onset (LEO) Team is a community team comprising 10 members of staff (team leader, part time consultant psychiatrist, trainee psychiatrist, half time clinical psychologist, occupational therapist, four community psychiatric nurses, and two healthcare assistants). It was established on the principles of assertive outreach, providing an extended hours service by including weekends and public holidays. ${ }^{2}$ Evidence based interventions adapted to the needs of people with early psychosis included low dose atypical antipsychotic regimens, cognitive behaviour therapy based on manualised protocols, ${ }^{17} 18$ and family counselling and vocational strategies based on established protocols. ${ }^{23}{ }^{24}$ Adherence to the assertive outreach model and to these treatment protocols was ensured through supervision of cognitive behaviour therapy (PG), medication prescribing, family support, and the assertive outreach model 
(PP and TKJC). Whereas medication was prescribed to all patients, the range of psychological interventions varied according to need as assessed by the treating clinicans.

\section{Standard care}

Patients in the control group received standard care delivered by the community mental health teams. These teams received no additional training in the management of early psychosis, although they were encouraged to follow available guidelines. ${ }^{23} 25$

\section{Assignment}

All referrals to the psychiatric services in Lambeth were screened for possible psychotic symptoms (SC, MF-A, and NR) ${ }^{26}$ Those passing this initial screen were interviewed by one researcher (NR) to confirm symptoms (using the schedules of clinical assessment in neuropsychiatry) and to establish the likely date of onset and previous contact with services. Finally each patient was assigned a provisional diagnosis. ${ }^{27}$ Basic demographic and personal data were recorded at this point.

Eligible patients were randomised to specialised care or standard care by permuted random blocks of between two and six. Group allocation was concealed in sealed envelopes. The trial statistician (GD) independently carried out the randomisation and concealment of results. Patients were informed of the randomisation process, and written consent was sought to collect outcome data from case notes and by interview (SC and MF-A) as soon as feasible after randomisation and at follow up 18 months later.

\section{Primary outcomes}

Our primary outcome measures were rates of relapse and readmission to hospital. Two of the researchers (TKJC and PG) agreed on the ratings for recovery (full or partial) and relapse, basing these ratings on operationalised criteria (available from authors) applied to extracts of the clinical case notes from which information pertaining to group allocation had been removed. Group allocation remained concealed until completion of the ratings. To test the success of blinding, assessors guessed the group allocation of each patient. The two raters correctly guessed the allocation of $60 \%(95 \%$ confidence interval $52 \%$ to $63 \%$ ) of the patients ( $\kappa 0.20$ ). Data on readmissions were gathered through the hospital administration system.

\section{Statistical analysis}

We determined that we required 120 patients. This was based on an estimate that $60 \%$ of patients in the control group and $40 \%$ of patients in the specialised care group would relapse at 18 months, at a power of $80 \%$ and an $\alpha$ of 0.05 . We also estimated that $25 \%$ of patients would drop out over the 18 months of follow up.

We analysed data using STATA release 7. Using intention to treat analyses we compared the outcomes in both groups at 18 months and determined whether patients had relapsed at any point. Patients who had previously relapsed but had recovered by 18 months were included as "well" at that point. Differences are reported as odds ratios and based on the $\chi^{2}$ statistic. Readmissions were similarly analysed. Further analyses compared the two groups for total number of readmissions across the follow up period, total duration of hospital stay, and the proportion of admissions that occurred under the mental health act. Subsequent analyses controlled for possible imbalances in characteristics at baseline. We used an analysis of covariance with logistic regression approaches for categorical data and regression for total number of admissions, readmissions, and

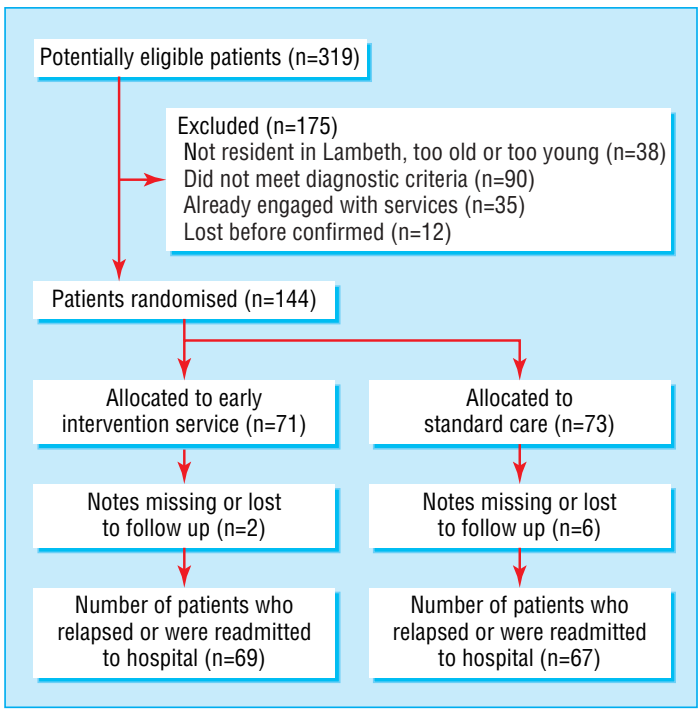

Flow of patients through trial

duration of hospital stay. Standard errors and confidence intervals were obtained through bootstrap methods.

We estimated the duration of untreated psychosis, defined as the period from the first onset of positive symptoms to initial contact with psychiatric services in patients for whom this was their first contact.

\section{Results}

Overall, 319 people presented to psychiatric services between January 2000 and October 2001 with symptoms suggestive of a psychotic disorder. Of these, 144 met the inclusion criteria and were randomised to receive either specialised care or standard care (figure). Data on number of relapses and readmissions to hospital were obtained for $136(94 \%)$ patients over the 18 months of follow up. We had complete information on clinical status (recovered, unwell or relapsed) for $131(91 \%)$ patients at 18 months.

Both groups were similar for most characteristics at baseline, although the specialised care group had fewer men, a higher proportion of first psychotic episodes, and a higher proportion of white people (table 1). Patients in the specialised care group also had a longer duration of untreated psychosis, although the difference between the groups was not statistically significant (mean (SD) duration (months) of untreated psychosis: 10.5 (17.2) for specialised care $v 7.6$ (10.7) for controls).

For most patients, admission to hospital was their first experience of mental health care (43 of 71 patients (61\%) in specialised care group, 44 of 73 patients $(60 \%)$ in control group) two thirds of which were involuntary admissions (specialised care $67 \%$, controls $72 \%)$.

At 18 months, one patient in the control group had died (unknown cause); 53 (86\%) patients in the specialised care group and $44(68 \%)$ patients in the control group were in regular contact with the clinical team (lost to care: odds ratio $0.35,95 \%$ confidence interval 0.15 to 0.81 ). Patients in the specialised care group were offered more appointments during follow up than controls (mean (SD) number of appointments: 17.4 (9.1) for specialised care $v 13.2$ (8.7) for controls) and failed to attend a smaller proportion of the appointments (mean (SD) proportion of appointments missed: $0.12(0.2)$ for specialised care $v 0.33$ (0.3) for controls). Seven patients in the specialised care group 
Table 1 Baseline characteristics of groups receiving specialised care or standard care for psychosis. Values are numbers (percentages) unless stated otherwise

\begin{tabular}{|c|c|c|}
\hline Characteristics & $\begin{array}{l}\text { Specialised care group } \\
\qquad(\mathrm{n}=71)\end{array}$ & $\begin{array}{l}\text { Standard care group } \\
\qquad(\mathrm{n}=73)\end{array}$ \\
\hline Mean (SD) age (years) & $26(6.0)$ & $26.6(6.4)$ \\
\hline Men & $39(55)$ & $54(74)$ \\
\hline First episode & $61(86)$ & $52(71)$ \\
\hline \multicolumn{3}{|l|}{ Ethnicity: } \\
\hline White & $27(38)$ & $18(25)$ \\
\hline Black British & $10(14)$ & $6(8)$ \\
\hline Black Caribbean & $9(13)$ & $13(18)$ \\
\hline Black African & $16(23)$ & $25(34)$ \\
\hline Mixed & $6(8)$ & $6(8)$ \\
\hline Other & $3(4)$ & $5(6)$ \\
\hline Single & $50(71)$ & $51(73)$ \\
\hline \multicolumn{3}{|l|}{ Living situation: } \\
\hline Family & $37(54)$ & $40(55)$ \\
\hline Alone & $23(33)$ & $18(25)$ \\
\hline Other ${ }^{*}$ & $9(13)$ & $15(20)$ \\
\hline \multicolumn{3}{|l|}{ Employment: } \\
\hline Full time & $9(13)$ & $8(11)$ \\
\hline Part time & $4(6)$ & $5(7)$ \\
\hline Unemployed & $45(63)$ & $45(64)$ \\
\hline Student & $10(14)$ & $10(14)$ \\
\hline Housewife & $3(4)$ & $2(3)$ \\
\hline Schizophrenia & $51(72)$ & $49(67)$ \\
\hline
\end{tabular}

* Shared with friends or living in hostel.

and 10 control patients were in hospital at the 18 months' follow up. One control patient was in prison.

Table 2 Contact with mental health services and uptake of treatment over 18 months for patients receiving care for early psychosis. Values are numbers (percentages) of patients unless stated otherwise

\begin{tabular}{|c|c|c|c|}
\hline Variable & $\begin{array}{l}\text { Specialised care group } \\
\qquad(\mathrm{n}=71)\end{array}$ & $\begin{array}{l}\text { Standard care } \\
\text { group }(n=73)\end{array}$ & $\begin{array}{l}\text { Odds ratio }(95 \% \\
\text { CI) }\end{array}$ \\
\hline $\begin{array}{l}\text { In contact with index } \\
\text { team }\end{array}$ & 54 (76) & $43(59)$ & 2.4 (1.2 to 4.9) \\
\hline $\begin{array}{l}\text { In contact with any } \\
\text { mental health service }\end{array}$ & $60(85)$ & 46 (63) & $2.9(1.2$ to 6.7$)$ \\
\hline \multicolumn{4}{|l|}{ Psychosocial treatment: } \\
\hline $\begin{array}{l}\text { Psychological } \\
\text { interventions }\end{array}$ & $39(55)$ & $20(27)$ & 3.2 (1.6 to 6.5$)$ \\
\hline Family interventions & $40(56)$ & $17(33)$ & 4.3 (2.1 to 8.7 ) \\
\hline $\begin{array}{l}\text { Vocational } \\
\text { interventions }\end{array}$ & $36(51)$ & $17(23)$ & 3.4 (1.6 to 6.9$)$ \\
\hline \multicolumn{4}{|l|}{ Uptake of intervention: } \\
\hline One & $18(25)$ & $34(47)$ & - \\
\hline Two & $29(41)$ & $6(8)$ & - \\
\hline All & $13(18)$ & $2(3)$ & - \\
\hline
\end{tabular}

Patients in the specialised care group were more likely than those in the control group to have been offered psychosocial interventions (table 2).

Patients in the specialised care group were significantly more likely to be in recovery at follow up than patients in the control group; this included patients who had relapsed (table 3). When only those patients who had not relapsed after initial recovery were classified as "well" (specialised care 64\%, controls 48\%), the difference between groups was attenuated and fell below statistical significance (odds ratio $0.52,95 \%$ confidence interval 0.26 to 1.03).

Most patients in both groups recovered from the index episode (specialised care: full recovery $71 \%$, partial recovery 19\%; controls: full recovery $63 \%$, partial recovery $28 \%$ ). The average time to recovery was 5.5 (SD 4.0) months in both groups. Although there were no differences in these rates, patients in the specialised care group were less likely to relapse than those in the control group (specialised care 30\% v controls 48\%; $\mathrm{P}=0.042$ ). Patients in the specialised care group were also readmitted fewer times during follow up (see table 3).

Although the higher contact with services among patients in the specialised care group remained significant (lost to care: odds ratio $0.28 ; 95 \%$ confidence interval 0.12 to 0.73 ) when we adjusted for baseline differences in sex, previous psychotic episodes, and ethnic minority group, only the difference in total number of readmissions during follow up remained statistically significant (see table 3).

\section{Discussion}

Patients with early psychosis who are cared for by a specialist team are more likely to maintain contact with psychiatric services and to have fewer readmissions to hospital than patients who receive standard care. This finding is, however, limited because our study was underpowered.

The strengths of our study are the use of randomisation, the low attrition for primary outcome data, and the inclusion of all patients with a first episode of non-affective psychosis presenting to secondary mental health services within a defined inner city area.

Our study does, however, have several limitations. We relied on record systems for data on relapse, which are susceptible to errors and biases. Our study is among the first, however, to use an a priori operational definition of recovery and relapse with clear definitions and a rating manual. Attempting to prospectively monitor symptom change by repeated interview is not only costly but also susceptible to differential rates of attrition, with a higher proportion of patients in the intervention group likely to remain in contact and willing to cooperate with interviews. That

Table 3 Primary outcome measures for patients receiving specialised care or standard care for early psychosis. Values are numbers (percentages) of patients unless stated otherwise

\begin{tabular}{|c|c|c|c|c|c|c|}
\hline Outcome & $\begin{array}{l}\text { Specialised care } \\
\text { group }\end{array}$ & Standard care group & $\begin{array}{l}\text { Regression coefficient or odds } \\
\text { ratio }(95 \% \mathrm{CI})\end{array}$ & $P$ value & $\begin{array}{l}\text { Adjusted regression coefficient } \\
\text { or odds ratio }(95 \% \mathrm{Cl})^{*}\end{array}$ & $P$ value \\
\hline $\begin{array}{l}\text { Full or partial recovery at } 18 \\
\text { months }\end{array}$ & $55 / 66$ (83) & $43 / 65(66)$ & $0.41(0.18$ to 0.94$)$ & 0.035 & $0.46(0.19$ to 1.12$)$ & 0.087 \\
\hline Recovery (full or partial) & $61 / 69(90)$ & $61 / 67(91)$ & 1.15 (0.36 to 3.61$)$ & 0.814 & $1.76(0.51$ to 6.09$)$ & 0.371 \\
\hline Relapse (full or partial) $\dagger$ & $18 / 61(30)$ & $29 / 61$ (48) & $0.46(0.21$ to 0.97$)$ & 0.042 & 0.55 (0.24 to 1.26$)$ & 0.157 \\
\hline Any readmission $\ddagger$ & $23 / 69$ (33) & $33 / 67 \quad(51)$ & 0.48 (0.24 to 0.97$)$ & 0.041 & 0.53 (0.26 to 1.12$)$ & 0.095 \\
\hline Mean (SD) No of readmissions & $0.4(0.7)$ & $0.8(1.0)$ & 0.39 (0.10 to 0.68$)$ & 0.010 & 0.36 (0.04 to 0.66$)$ & 0.030 \\
\hline $\begin{array}{l}\text { Mean (SD) No of bed days in } \\
\text { follow up }\end{array}$ & $35.5(78.9)$ & $54.9(93.6)$ & 19.4 (-10.6 to 48.6) & 0.197 & $20.7(-10.9$ to 55.8$)$ & \\
\hline
\end{tabular}

${ }^{*}$ Adjusted for sex, past episode, and ethnicity.

$\dagger$ Excludes those who never recovered.

$\ddagger$ Regardless of whether initially recovered. 


\section{What is already known on this topic}

Outcomes for certain psychoses may be improved by early detection and maintenance of treatment for two or three years after onset

This ideal is seldom achieved by general psychiatric services What this study adds

Patients receiving specialised care for early psychosis maintained better contact with services and had fewer readmissions to hospital than patients receiving standard care

The data are limited, however, owing to the small sample size

there were fewer readmissions to hospital in patients receiving specialised care is encouraging, as this outcome is closely linked with relapse and outside the control of the research team.

Despite our efforts to include all first presentations and to minimise the number of people who dropped out, our study was underpowered. If we had attempted to address the issue of multiple primary outcomes (for example, by Bonferroni correction) the findings would not be statistically significant. Also, probably reflecting the modest sample size, the randomisation process did not produce well matched groups, with the specialised care group at baseline having more features of better prognosis for sex, previous psychotic episodes, and ethnicity than the standard care group. This is reflected in the adjusted analysis where only one of the six primary outcomes retained statistical significance.

It might be argued that our results are attributable not so much to the particular benefits of specialist provision of early intervention but to the comparison of such a service with inadequately resourced routine services. ${ }^{28}$ To some extent this is true, as an earlier study reported that disengagement from routine community services was common.$^{29}$ It has, however, been an empirical question as to whether establishing a new service would be acceptable to patients and lead to improved outcomes. Finally, it might also be said that our study has been overtaken by the UK's Department of Health initiative to establish 50 new early intervention services, but in practice there are wide variations in implementation with little guidance for service developers on whether they should put the modest increase in funding towards early detection, home treatment, or, as in our case, efforts to prevent relapse.

We thank the clinical teams for access to participants and the patients and their carers.

Contributors: TKJC was responsible for the study conception and design, interpretation of the data, and writing the paper. He is the guarantor. PAC was responsible for the study conception and design and interpretation of the data. PP was the clinical lead of the specialised service and ensured that best practice guidelines were followed to early psychosis model. NR carried out the screening and clinical intake assessments and contributed to the study design, execution, and interpretation. SC contributed to the study execution, analysis, and interpretation. MF-A contributed to the execution of the study and analysis and interpretation of the data. GD was responsible for designing and overseeing the randomisation process, carried out the main statistical analysis, and contributed to the interpretation of the data and writing the paper.

Funding: Directorate of Health and Social Care London research and development organisation and management programme (grant No Brixton Early Psychosis Project RDC 01657). The views expressed are those of the authors and not necessarily those of the NHS or the Department of Health.
Competing interests: TKJC and PP have received support for attending seminars and fees for speaking at conferences on early psychosis from Eli Lilly, AstraZeneca, Janssen-Cilag, and Novartis.

Ethical approval: This study was approved by the local research ethics committee.

1 Department of Health. The NHS plan. A plan for investment. A plan for reform. London: DoH, 2000.

2 Department of Health. The mental health policy implementation guide. London: $\mathrm{DoH}$, 2001.

3 Loebel AD, Lieberman JA, Alvir JM, Mayerhoff DI, Geisler SH, Symanski SR. Duration of untreated psychosis and cognitive functioning in first episode patients. Am J Psychiatry 1992;149:1183-8.

4 McGorry PD, Edwards J, Mihalopoulos C, Harrigan SM, Jackson HJ. EPPIC: an evolving system of early detection and optimal management. Schizophr Bull 1996;22:305-26. 5 Wyatt RJ, Green MF, Tuma AH. Long term morbidity associated with delayed treatment of first admission schizophrenic patients. Psychol Med 1997;27:261-8.

6 Drake RJ, Haley CJ, Akhtar S, Lewis S. Causes and consequences of duration of untreated psychosis in schizophrenia. Br J Psychiatry 2000;177:511-5.

7 Birchwood M, Todd P, Jackson C. Early intervention in psychosis: the critical period hypothesis. BrJ Psychiatry 1998;172(Suppl 33):53-9.

8 Melle I, Larsen TK, Haahr U, Friis S, Johannessen JO, Opjordsmoen S, et al. Reducing the duration of untreated first-episode psychosis: effects on clinical presentation. Arch Gen Psychiatry 2004;61:143-50.

9 Drury V, Birchwood M, Cochrane R, Macmillan F. Cognitive therapy and recovery from acute psychosis: a controlled trial. I. Impact on psychotic symptoms. Br J Psychiatry 1996:169:593-601.

10 Birchwood M, McGorry P, Jackson H. Early intervention in schizophrenia. Br J Psychiatry 1997;170:2-5.

11 Nordentoft M, Jeppesen P, Abel M, Kassow P, Petersen L, Thorup A, et al. OPUS study: suicidal behaviour, suicidal ideation and hopelessness among patients with first-episode psychosis: one-year follow-up of a randomised controlled trial. $\mathrm{Br} J$ Psychiatry 2002;181(Suppl 43):98-106.

12 Malla A, Norman R, McLean T, Scholten D, Townsend L. A Canadian programme for early intervention in non-affective psychotic disorders. Aust NZ J Psychiatry 2003:37:407-13.

13 Addington J. Leriger E, Addington D. Symptom outcome 1 year after admission to an early psychosis program. Can J Psychiatry 2003;48:204-7.

14 Cullberg J, Levander S, Holmqvist R, Mattson M, Wieselgren I. One year outcome in first episode psychosis patients in the Swedish parachute project. Acta Psychiatr Scand 2002;106:276-85.

15 Malla AK, Norman RM, Scholten DJ, Zirul S, Kotteda V. A comparison of long-term outcome in first-episode schizophrenia following treatment with risperidone or a typical antipsychotic. J Clin Psychiatry 2001;62:179-84.

16 Lieberman JA, Tollefson G, Tohen M, Green AI, Gur RE, Kahn R, et al. Comparative efficacy and safety of atypical and conventional antipsychotic drugs in first-episode psychosis: a randomized, double-blind trial of olanzapine versus haloperidol. Am J Psychiatry 2003;160:1396-404

17 Jolley S, Garety P, Craig T, Dunn G, White J, Aitken M. Cognitive therapy in early psychosis: a pilot randomised controlled trial. Behav Cog Psychother 2003;31:473-8.

18 Jackson H, McGorry P, Edwards J, Hulbert C, Henry L, Francey S, et al. Cognitively orientated psychotherapy for early psychosis (COPE). Br J Psychiatry 1998;172(Suppl 33): $93-100$

19 Lewis S, Tarrier N, Haddock G, Bentall R, Kinderman P, Kingdon D, et al. Randomised controlled trial of cognitive-behavioural therapy in early schizophrenia: acute-phase outcomes. Br J Psychiatry 2002;181(Suppl 43):91-7.

20 Linszen D, Dingemans P, Van der Does JW, Nugter A, Scholte P, Lenior R, et al. Treatment, expressed emotion and relapse in recent onset schizophrenic disorders. Psychol Med 1996;26:333-42.

21 Zhang M, Wang M, Lee J, Phillips MR. Randomised control trial of family intervention for 78 first-episode male schizophrenic patients: an 18 month study in Suzhou, Jiangsu. BrJ Psychiatry 1994:165(Suppl 24):96-102.

22 Department of Health. Compendium of clinical and social indicators. London: $\mathrm{DoH}, 2001$.
23 Early Psychosis Prevention and Intervention Centre. EPPIC early psychosis training pack. Melbourne: EPPIC, 1997.

24 Edwards J, McGorry PD. Implementing early intervention in psychosis: a guide to establishing early psychosis services. London: Martin Dunitz, 2002.

25 Aitchison K, Meehan K, Murray RM. First episode psychosis. London: Martin Dunitz, 1999.

26 Jablensky A, Sartorius N, Ernberg G, Anker M, Korten A, Cooper JE, et al. Schizophrenia:manifestations, incidence and course in different cultures. A World Health Organization ten country study. Cambridge: Cambridge University Press, 1992.

27 McGuffin P, Farmer A, Harvey I. A polydiagnostic application of operational criteria in studies in psychotic illness: development and reliability of the OPCRIT system. Arch Gen Psychiatry 1991;48:764-70.

28 Pelosi A, Birchwood M. Debate: is early intervention for psychosis a waste of valuable resources? Br J Psychiatry 2003;182:196-8.

29 Garety PA, Rigg A. Early psychosis in the inner city: a survey to inform service planning. Soc Psychiatry Psychiatr Epidemiol 2001;36:1-8. (Accepted 4 August 2004)

doi $10.1136 /$ bmj.38246.594873.7C

Institute of Psychiatry, De Crespigny Park, London SE5 8AF

Tom K J Craig professor of social and community psychiatry

Philippa Garety professor of psychology

Susannah Colbert research assistant

Miriam Fornells-Ambrojo research assistant

Lambeth Early Onset Service, South London and Maudsley NHS Trust, London SW9 9NT 
Paddy Power consultant psychiatrist

Nikola Rahaman specialist registrar

Biostatistics Group, School of Epidemiology and Health Science, University of
Manchester, Manchester M13 9PT

Graham Dunn professor of biomedical statistics

Correspondence to: T K J Craig t.craig@iop.kcl.ac.uk 